\title{
Inferring of Cognitive Skill Zones in Concept Space of Knowledge Assessment
}

\author{
Rania Aboalela \\ Kent State University \\ Kent, Ohio, U.S.A
}

\author{
Javed Khan \\ Kent State University \\ Kent, Ohio, U.S.A
}

\begin{abstract}
In these research zones of the knowledge, the assessed domain is identified. Explicitly, these zones are known as Verified Skills, Derived Skills and Potential Skills. In detail, the Verified Skills Zone is the set of tested concepts in the knowledge domain, while Derived Skills Zone is the set of the prerequisite concepts to the tested concepts based on the cognitive skills relation, whereas Potential Skills Zone is the set in the domain that have never been tested or prerequisite to the tested concepts but they are related to the tested concept based on the cognitive relation skills. Identifying cognitive relations between the concepts in one domain simplifies the structure of the assessment, which helps to find the knowledge state of the assessed individual in a short time and minimum number of questions. The existence of the concepts in the assessment domain helps us to estimate the set of the concepts that are known or not known or ready to be known or not ready to be known. In addition, it provides the output of the assessment in concept centric values in addition to the quantity values. The assessment result gives binary values of the assessed domain. " 1 " implies knowing the concept, whereas " 0 " implies not knowing the concept. The output is six sets of concepts: 1) Verified Known Skills; 2) Verified Not Known Skills; 3) Derived Known Skills; 4) Derived Not Known Skills; 5) Potential Known Skills; and 6) Potential Not Known Skills. The experiment is conducted to show the binary output of the assessed domain based on the participants' answers to the asked questions. The results also highlight the efficiency of the assessment.
\end{abstract}

Keywords-Cognitive skill; bloom's taxonomy; assessment of knowledge; concept space; concepts zones

\section{INTRODUCTION}

The knowledge assessments of knowledge space [1] requires large number of questions and the assessment structure is complicated. They don't identify cognitive difficulty variations of learning. The previous research work applied the assessment of knowledge in one space such as [1], [2] are only applicable for a domain that has clear relations like Mathematical Fields. Applied science fields like Software Engineering and Medical Science need identifying internal cognitive relations to simplify the assessment structure and to give an accurate assessment result. The difficulty in assessing the knowledge in one domain studied by [3] introduced ontological relation between the concepts in the course and test questions. In addition, the research work [4] proposed a skill that can be characterized as a pair consisting of a concept and an activity. As an example of such a pair, they give "Apply the Pythagorean Theorem". In this study, the researchers concentrate on the concepts as they appear in the text in either phrase form or single word form and identify the link between the concepts, as the skill required to learn the concept at a certain skill level, which identifies the prerequisite relation between the concepts. Moreover, [5], [6] studied and validated the efficiency and importance using the parameter of cognitive skill level to assess the knowledge in one domain. The research work [7] introduce a model of the cognitive level relation between the concepts in the learning assessment. The cognitive skill that used is the verbs of the Revised Bloom's Taxonomy. The revised version identified six verbs infer to six categories of skills. The original taxonomy was created in 1956 by Dr. Benjamin Bloom [8]. The Blooms' Taxonomy arranges what the learner has to learn in a hierarchy of six levels. In 2001, the six major categories were changed from noun to verb forms and renamed [9]. In this research, the verbs of the revised Blooms' Taxonomy used to identify the prerequisite cognitive relation between the concepts in the domain. The six verbs are inferred using skill numbers that indicate the cognitive difficulty as the following:

"1" means recall, "2" means understanding, "3" means applying, "4" means analyzing, "5" means evaluating and "6" means creating. The researchers in this research work use the five higher levels in referring to the level needed to acquire the concept at the cognitive skill levels of understanding, applying, analyzing, or creating the concepts.

The present study is structured as follows: materials and methods in Sections II and III. The complexity of assessing the knowledge in one domain is discussed in Section IV. The component of the assessment is discussed in Section V. The experimental results in Section VI. The conclusion is given in Section VII. The discussion and future work is discussed in Section VIII.

\section{COGNITIVE SKILl ZoNeS}

\section{A. The Concept Zones of Verified Skills}

First, Verified Skills zone is the zone of the main concepts that have to be tested and they infer to the most concepts in the assessed domain. VS is defined as where there is a direct evidence that a learner knows concept $\mathrm{C}_{\mathrm{x}}$ at a cognitive skills level $\mathrm{k}$ it is considered to belong to verified set VS(k). To illustrate the VS, let us consider a question $\mathrm{Q}_{i}$, which can ascertain that a student knows a specific concept $\mathrm{C}_{\mathrm{x}}$. The verified skills satisfy the condition that:

If $\left(\mathrm{Q}_{\mathrm{i}}, \mathrm{C}_{\mathrm{x}}\right) \mathrm{L}_{\mathrm{k}} \& \mathrm{C}_{\mathrm{x}}$ is correct answer $\rightarrow \mathrm{C}_{\mathrm{x}} \in \mathrm{VS}(\mathrm{k}) \forall \mathrm{C}_{\mathrm{x}} \in$ completely correct answer concepts. 


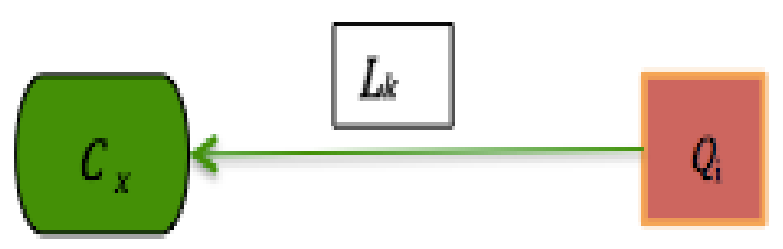

Fig. 1. Verified Skills (VS) Zone.

Where, $\mathrm{Q}_{\mathrm{i}} \in$ Test questions, $\mathrm{C}_{\mathrm{x}} \in$ Tested concepts, $\mathrm{L}_{\mathrm{k}} \in$ Bloom link of level $\mathrm{k}, \mathrm{VS}(\mathrm{k}) \in$ Verified skills at level $\mathrm{K}$ and $\left(\mathrm{Q}_{\mathrm{i}}, \mathrm{C}_{\mathrm{x}}\right) \mathrm{L}_{\mathrm{k}}$ means existing link between the question $\mathrm{Q}_{\mathrm{i}}$ and the concept $\mathrm{C}_{\mathrm{x}}$ at level $\mathrm{K}$. The link means that to answer $\mathrm{Q}_{\mathrm{i}}$ correctly $\mathrm{C}_{\mathrm{x}}$ must be learned at level k. Fig. 1 shows verified skill link.

\section{B. Concept Zones of Derived Skills at Level 2, DS $(K=2)$}

The Zones of the derived skills is the set of the concepts that must be understood to attain learning the VS. Derived Skill is defined as where there is indirect evidence that a student knows a concept $C_{i}$ at a cognitive skills level 2 (the understand level), it is considered to be a part of DS (2). In other words, if there is indirect evidence that the concept $\mathrm{C}_{\mathrm{i}}$ is understood by the student then it will belong to DS (K=2). The condition of the relation is expressed as the following:

If $\mathrm{C}_{\mathrm{i}}$ is not a member in a verified set but there exists two links such that $\left(\mathrm{Q}_{\mathrm{i}}, \mathrm{C}_{\mathrm{x}}\right) \mathrm{L}_{\mathrm{k}},\left(\mathrm{C}_{\mathrm{i}}, \mathrm{C}_{\mathrm{x}}\right) \mathrm{L}_{\mathrm{m}}$ \& $\mathrm{C}_{\mathrm{x}} \in \mathrm{VS}(\mathrm{k})$ that it is a member in VS and $m=2$ and $\mathrm{k}>=\mathrm{m}$, then $\mathrm{C}_{\mathrm{i}}$ is a member in DS at level 2, i.e. $C_{i} \in D S(2), Q_{i} \in$ Test Questions. $C_{x} \in V S$, $\mathrm{C}_{\mathrm{i}} \in$ another concept in the concept space. The $\left(\mathrm{Q}_{\mathrm{i}}, \mathrm{C}_{\mathrm{x}}\right) \mathrm{L}_{\mathrm{k}}$, $\left(\mathrm{Q}_{\mathrm{i}}, \mathrm{C}_{\mathrm{x}}\right) \mathrm{L}_{\mathrm{m}}$ means existing link between the Question $\mathrm{Q}_{\mathrm{i}}$ and the Concept $\mathrm{C}_{\mathrm{x}}$ at level $\mathrm{k}$ and $\mathrm{m}$ respectively. Fig. 2 illustrates DS relation at level 2.

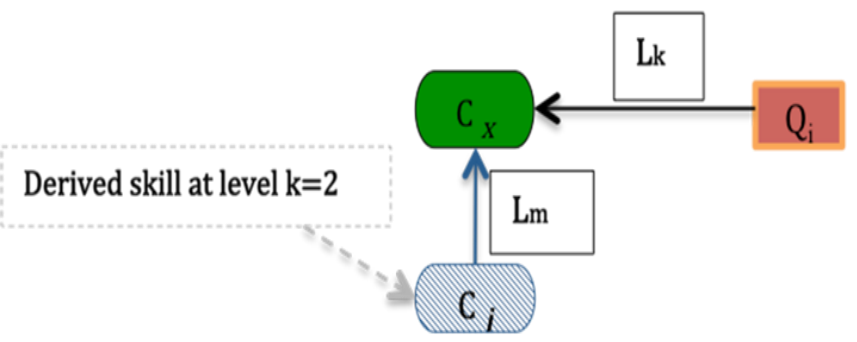

Fig. 2. Derived Skill (DS) Zone at level 2.

\section{Zones of the Support Set (SS)}

To distinguish the higher-level relations, the classification of the set into Support Set and Supported Set must be identified. The support set means the prerequisite set of the supported set. Let $\mathrm{C}_{\mathrm{A}}$ be a node. Let $\mathrm{C}_{\mathrm{B}}$ be another node from where there is a level $\mathrm{k}$ link to $\mathrm{A}$. Then $\mathrm{C}_{\mathrm{B}}$ at level $\mathrm{k}$ is called the support node of $C_{A}$. That means $C_{B}$ is the prerequisite set of $\mathrm{C}_{\mathrm{A}}$ concept at level $\mathrm{k}$. Let $\mathrm{S}\left(\mathrm{C}_{\mathrm{A}}, \mathrm{k}\right)$ be the set of all such $\mathrm{C}_{\mathrm{B}}$ nodes in the complete concept graph $\mathrm{G}$. The $\mathrm{S}\left(\mathrm{C}_{\mathrm{A}}, \mathrm{k}\right)$ is the level $\mathrm{k}$ Support Set for $\mathrm{C}_{\mathrm{A}}$. i.e. all concepts in this set must be learned to have a level $\mathrm{k}$ skill in A. Fig. 3 illustrates the Support Set \& Support Node, which is any node in the Support Set.

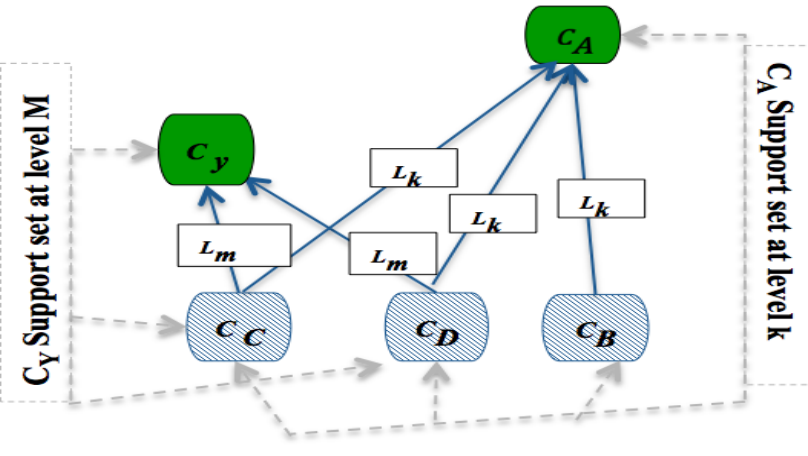

Fig. 3. Support Node (SN) \& Support Set (SS).

\section{Zones of Derived Skill, DS $(k>2)$}

DS $(k>2)$ means that there is a direct evidence a learner knows a concept $\mathrm{C}_{\mathrm{y}}$ at a cognitive skill level 2, and there is indirect evidence he knows it at a cognitive skill level higher than a cognitive skill level 2. In other words, by inference a learner could either apply/analyze/evaluate/create, a concept $\mathrm{C}_{\mathrm{y}}$. The relation condition is illustrated as the following: If $\mathrm{C}_{\mathrm{y}}$ is known i.e. it is in $\mathrm{DS}(2)$ or $\mathrm{VS}(2)$, and if all level $\mathrm{k}$ support nodes of $C_{y}$ i.e. $S\left(C_{y}, k\right)$ is in $\operatorname{VS}(2) \operatorname{VDS}(2)$, then $C_{y}$ will be considered as a Derived Skill at level k. In other words. If $\mathrm{C}_{\mathrm{y}} \in$ $\mathrm{DS}(2) \vee \mathrm{VS}(2)$ and $\mathrm{S}\left(\mathrm{C}_{\mathrm{y}}, \mathrm{k}\right)$ is subset of $\mathrm{DS}(2) \vee \mathrm{VS}(2)$ $\rightarrow \mathrm{C}_{\mathrm{y}} \in$ DS(k). Fig. 4 illustrates DS (k>2).

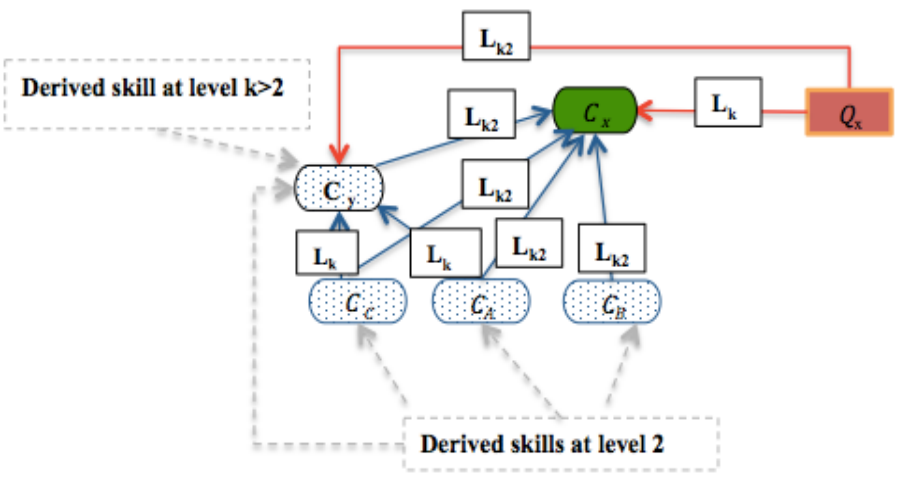

Fig. 4. Derived Skills relation at level $>2$.

\section{E. Zones of Potential Skill PS [( $k>2)]$}

The zones of Potential Skills $(k>2)$ is defined as where there is indirect evidence that a learner knows a particular concept such as "A" at a cognitive skills level higher than 2 (apply/ analyze/ evaluate/ create), it is a part of PS ( $\mathrm{k}>2)$. The concepts in the Zone PS have never been tested but their prerequisite concepts at the target skills level are tested either directly or indirectly. The relation condition is illustrated as the following: Let $S(A, k)$ is the support set of $A$ at level $k$. If every node in the $\mathrm{S}(\mathrm{A}, \mathrm{k})$ is subset of VS $\mathrm{V}$ DS at any level (doesn't matter-because The study only want to guarantee that the set is known) i.e. $\mathrm{S}(\mathrm{A}, \mathrm{k}) \subset \mathrm{VS}() \vee \mathrm{DS}()$, but there is no evidence that $A$ is known, then $A$ is in potential skill set $P S(k)$ i.e. $A \in P S(k)$, where $C_{d}, C_{x} \in(V S)$ and $C_{C}, C_{A}, C_{B} \in(D S)$ and $\mathrm{L}_{\mathrm{k}} \in$ Bloom's link at level k. Fig. 5 illustrates Potential Skills relation. 


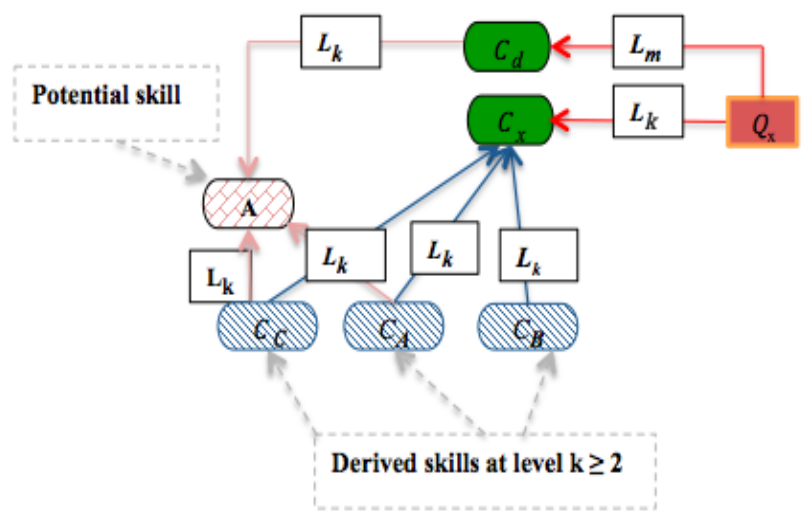

Fig. 5. Potential skills zone.

\section{Concept MapPed Testing AND Evaluation Method}

To measure the student learning, a concept mapped testing and evaluation method is set up. A test is composed of a set of questions. The learners are required to answer the questions based on their knowledge. Grader evaluates the student knowledge based on the answers. In conventional evaluation, a grader grades the answers and assigns a quantitative score for the student. The researchers slightly modify the evaluation method where the grader instead of a numerical score, is asked to evaluate if there is evidence in the answer that the student has succeeded or failed to attain learn a concept at a certain cognitive skill level. The researchers called it concept mapped testing \& evaluation method. Each tested concept in the assessment domain is labeled to the question based on the cognitive level as the following theory: To answer the question $\mathrm{Q}_{\mathrm{i}}$ correctly the concept $\mathrm{C}_{\mathrm{x}}$ must be known at skill level Lk. Fig. 6 shows the relation.

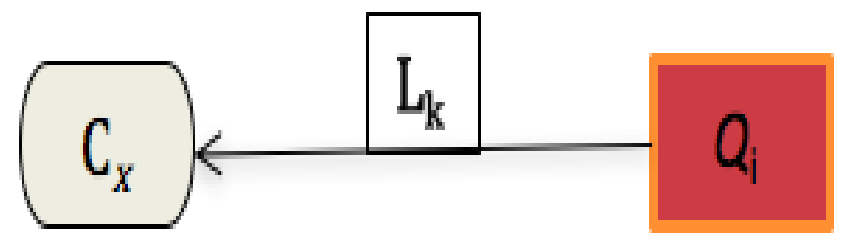

Fig. 6. The theory of connect the question to the tested concept.

\section{THE COMPLEXIITY OF ACCESSISING THE KNOWLEDGE IN ONE DOMAIN}

Assessing the knowledge in one domain is complex and requires large number of questions to ask about the target skill level of each concept in the domain. It is hard to capture a question for each concept in the domain at the target skill level. To solve this problem, assessment zones to classify which concepts should be directly tested or indirectly tested were obtained. Zones of the assessment were identified to simplify and decrease the number of the tested concepts. In this study, the researchers propose making the link between any two concepts a verb of the skill which needs to be learned, for example: to "apply" a concept B, the individual must know the prerequisite concept A at skill level 2, which is the understanding level. The "apply" is indicated by the number 3 in the link. Once the proposed zones are considered then the number of questions would be minimized to test only the concepts in the VS zones. Visualizing the concepts of one domain by using cognitive level mapped concepts graphs accomplished in [9]. The idea of automatically discovering and extracting the Bloom's Taxonomy from the text in one knowledge space is studied by Nafa, Khan and their colleagues [10]-[12].

\section{ASSESSMENT COMPONENTS}

\section{A. The Input of the Assessment}

The input is composed of:

1) The assessment domain graph, which is the cognitive skill level mapped concept graph of the assessed domain. The concepts mapped together with the prerequisite relation based on the cognitive skills.

2) The set of the questions mapped with the concepts in the concepts space.

3) The set of learners' responses to the set of questions.

\section{B. The Output of the Assessment}

The assessment goal is to find the knowing concepts infered by " 1 " and the not knowing concepts infered by " 0 " in the zones of the concepts (which concepts are known and not in known in which zone). This is the binary of knowledge (learning states) of these concepts in the various zones. The learning states are six states of knowing and not knowing the concepts in the concepts zones. 1) Verified Known Skills, 2) Verified Not Known Skills, 3) Derived Known Skills, 4) Derived Not Known Skills, and 5) Potential Known Skills, 6) Potential Not Known Skills. The sets of known zones of VKS include such a concept whose question is answered correctly and given the value 1 . The sets of known zones of DKS include such a concept has never been tested but it is prerequisite to the concept in VKS. Each estimated concept in DKS is given the value 1 . The set of PKS zone, which they have never been tested but all their prerequisite concepts at a certain skill level are tested and given the result that they all are known. Thus, the concepts are considered ready to be known PKS. Each estimated concept in PKS is given the value 1. The set of PNS zone, which they have never been tested but all their prerequisite concepts at a certain skill level are tested and given the result that they all are not known. Thus, the concepts are considered not ready to be known PNS. Each estimated concept in PNS is given the value 1.

\section{EXPERIMENT}

An experiment is organized to prove the efficiency of identifying the relations of the cognitive skill level between the concepts to maximize the estimation of measurement the concepts from few tested concepts.

\section{A. The Experiment Setup}

A human subject test is organized to prove the efficiency of the methods. The test is composed of 9 questions, which are selected from the midterm questions that have been given to the learners by the instructor of the class. The class is CS 61002 Algorithms and Programming in the Computer Science department. The test was introduced online in one session. The 
participants are 154 graduate students, attending the class. In this setup, the questions are specially redesigned to directly test a certain skill level of each concept belonging to the assessment domain. Nine questions are asked about 18 concepts at certain skill levels. Thus the 18 concepts are in the zone of VS. The concepts in the zones DS and PS are not tested but their evaluation of their binary states values is estimated. This experiment proves that using the proposed methods associated with the cognitive relations optimizes the knowledge assessment. The result of the evaluation of the perfect learner shows that the amount of the estimated knowledge of the assessed learner could be increased by at least 3 times over the conventional assessment which uses just numerical methods. The perfect learner's answers are used to calculate the experiment footprint and the size of footprint of each relation method. Table I and Fig. 7 show the size of footprint according to the perfect learner. Footprint is the number of fundamental tested concepts. The perfect learner is the student who gives a correct answer to all the asked questions. As evident the size of VS footprint is 18, the size of DS footprint is 31 and the size of PS footprint is 31 , which means that if the learner answered the questions correctly, then it would tell he knows certain levels of each of CONCEEPTS AT EACH SKIL knowledge assessment methods with the cognitive relation, one can maximize the amount of the estimation knowledge of the assessed learners.

TABLE I. THE NUMBER OF TESTED AND ESTIMATED CONCEPTS AT EACH SKILl LEVEL IN THE VARIOUS ZONES VS, DS AND PS

\begin{tabular}{|l|l|l|l|}
\hline Skill Level & Verified & Derived & Potential \\
& VS & DS & PS \\
\hline L2 & 7 & 12 & 13 \\
\hline L3 & 4 & 11 & 11 \\
\hline L4 & 2 & 3 & 2 \\
\hline L5 & 1 & 2 & 2 \\
\hline L6 & 4 & 3 & 3 \\
\hline Sum & 18 & 31 & 31 \\
\hline
\end{tabular}

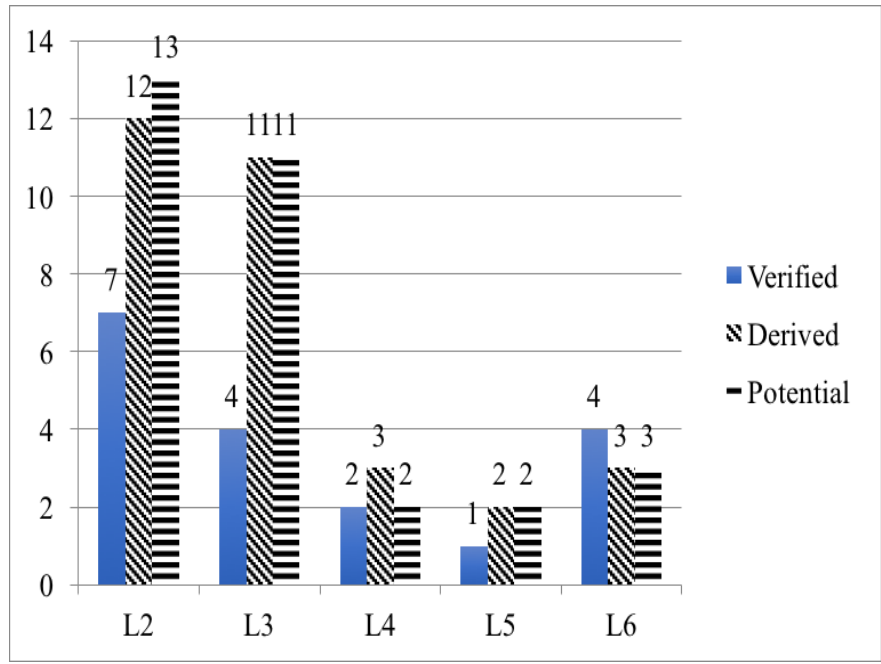

Fig. 7. The number of tested and estimated concepts at each skill level in the Various Zones VS, DS and PS.

\section{B. The Binary Concept States of the Human Subject Test}

The experiment is conducted to find out the binary set of 154 participants. Each participant of the 154 participants is assigned to number. Two participants we rechosen to show their binary concept states. One of them is the perfect student who gets all the answer correctly. The perfect students assigned to number 1 and the second student assigned to number 23. Also, each concept in the concept space is assigned to an integer number. The researchers show the binary concept state for the three zones VS, DS and PS. Thus, six concept zones are illustrated for the two participants. Fig. 8, 9 and 10 show the binary concept states of the 31 concepts in the zone of VS, DS and PS of the perfect student respectively. Fig. 11, 12 and 13 show the binary concept states of the 31 concepts in the zone of VS, DS and PS of the of the laziest student \#23 respectively.

\section{CONCLUSION}

The concept zones of the assessed domain are proposed. The efficiency of identifying cognitive relations between the concepts in the assessed domain is proved. Using the cognitive skills relation between the concepts in the assessment increases the amount of the estimated concepts, even though the number of tested concepts may be minimized and eliminated under the conditions laid down by the target cognitive skill levels. The binary concept state is assigned to the participants and the estimated binary concept states of the untested concepts are concluded.

\section{DISCUSSION AND FUTURE WORK}

There are many evidences or references that can infer the target skills of the concepts in the learning states zones. Sometimes many questions would be asked about the concepts in the domain and there can be a contradiction between the answers given. Also, errors might arise in the estimation of the learning states. In real exams, there can be other phenomena like lucky guess or careless mistakes. People have variant levels of initial knowledge. Accordingly, the probability computation should be used. This fact would be studied in the future work. The probability of the concepts states based on the cognitive relation of the concepts zones would be analyzed.

\section{REFERENCES}

[1] J.-C. Falmagne, E. Cosyn, J.-P. Doignon, and N. Thiery, "The assessment of knowledge, in theory and in practice ," in International Conference on Integration of Knowledge Intensive Multi-Agent Systems, pp. 609-615, 2003.

[2] J. C Falmagne and J. P. Doignon, Knowledge Spaces, Berlin, Springer, 1999.

[3] J. Khan, M. Hardas, Y. Ma, "A Study of problem difficulty evaluation for Semantic Network Ontology based intelligent courseware sharing," IEEE/WIC/ACM International Conference on Web Intelligence, WEB Intellegence, pp. 426-429, 2005.

[4] Heller, J., Steiner, C., Hockemeyer, C. \& Albert, D. “Competence-Based Knowledge Structures for Personalised Learning.," International Journal on E-Learning: Association for the Advancement of Computing in Education (AACE), 5(1), Chesapeake. VA, pp. 75-88, 2006.

[5] R. Aboalela \& J. Khan, "Are we asking the right questions to grade our students in a knowledge-state space analysis?," In IEEE 8th International Conference on Technology for Education (T4E), Mumbai, pp. 144-147, 2016. 
[6] R. Aboalela \& J. Khan, "Model of Learning Assessment to Measure Student Learning: Inferring of Concept State of Cognitive Skill Level in Concept Space," in In proceeding of the 3rd International Conference on Soft Computing \& Machine Intelligence (ISCMI), Dubai, United Arab Emirates, pp. 189-195. doi: 10.1109/ISCMI.2016.26

[7] B.S. Bloom, M.D. Engelhart, E.J. Furst, W.H. Hill, \& D.R. Krathwohl, (Eds.) Taxonomy of Educational Objectives. The Classification of Educational Goals, Handbook I: Cognitive Domain. David McKay Company, New York, 1956.

[8] L.W. Anderson, D.R. Krathwohl, P. W. Airasian, K.A. Cruikshank, R.E Mayer, P.R. Pintrich, et al., A taxonomy for learning, teaching, and assessing: A revision of Bloom's taxonomy of educational objectives. New York, U.S.A, Longman, 2001.

[9] R. Aboalela, J. Khan, "Visualizing concept space of course content," IEEE 7th International Conference on Engineering Education (ICEED).
Japan, November $2015 . \quad$ pp.

$609-615$ DOI: $10.1109 /$ ICEED 2015.7451512

[10] F. Nafa, \& , J. Khan "Conceptualize the Domain Knowledge Space in the Light of Cognitive Skills," In Proceedings of the 7th International Conference on Computer Supported Education. SCITEPRESS-Science and Technology Publications, pp. 285-295, 2015.

[11] F. Nafa, J. Khan, S.Othman, \& A. Babour, "Discovering Bloom Taxonomic Relationships between Knowledge Units Using Semantic Graph Triangularity Mining," International Conference on CyberEnabled Distributed Computing and Knowledge Discovery (CyberC), Chengdu: IEEE, pp. 224-233, 2016.

[12] F. Nafa, J.Khan, S. Othman, \& A. Babour "Mining Cognitive Skills Levels of Knowledge Units in Text Using Graph Tringluarity Mining," Web Intelligence Workshops (WIW), International Conference (IEEE/WIC/ACM), IEEE, pp. 1-4, 2016.

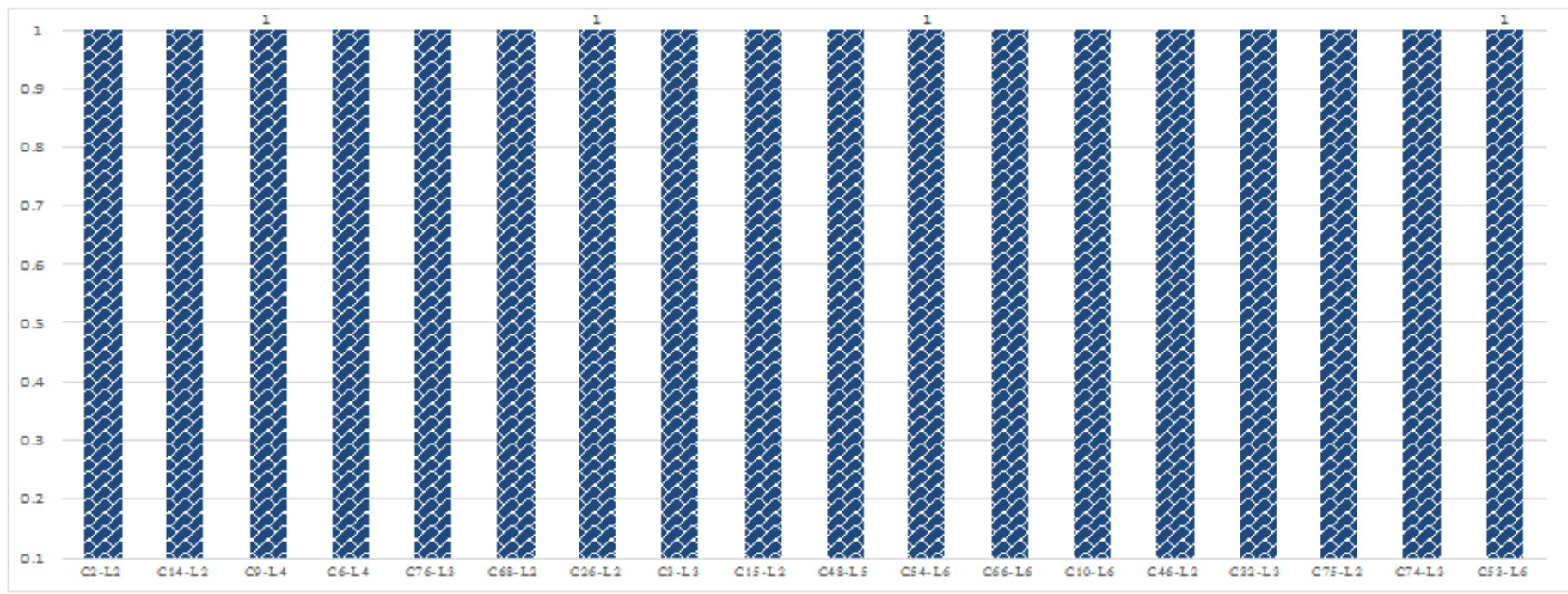

X: Concept Numbe

Y: Binary Number, "1" the concept is known. " 0 " The concept is unknown

Fig. 8. The VKS and VNS Zones of perfect learner.

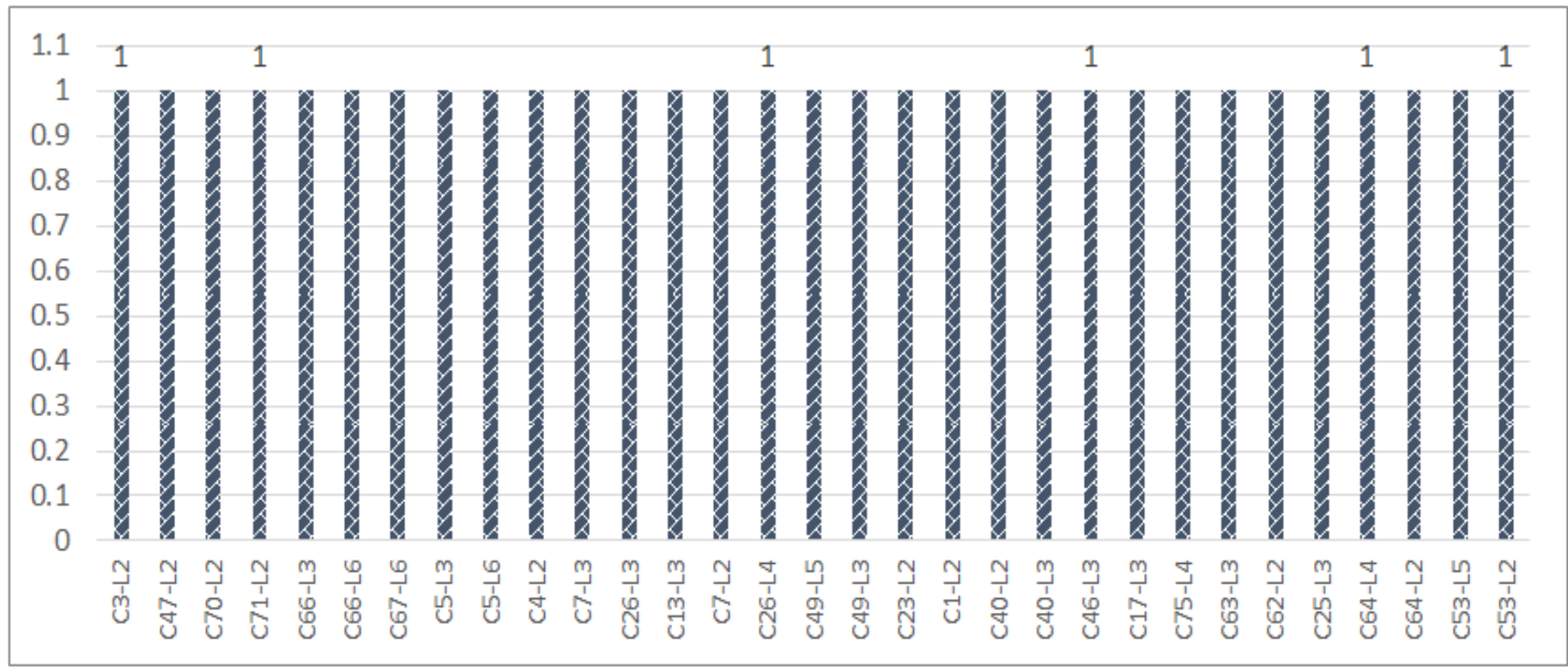

Fig. 9. The DKS and DNS Zones of perfect learner. 


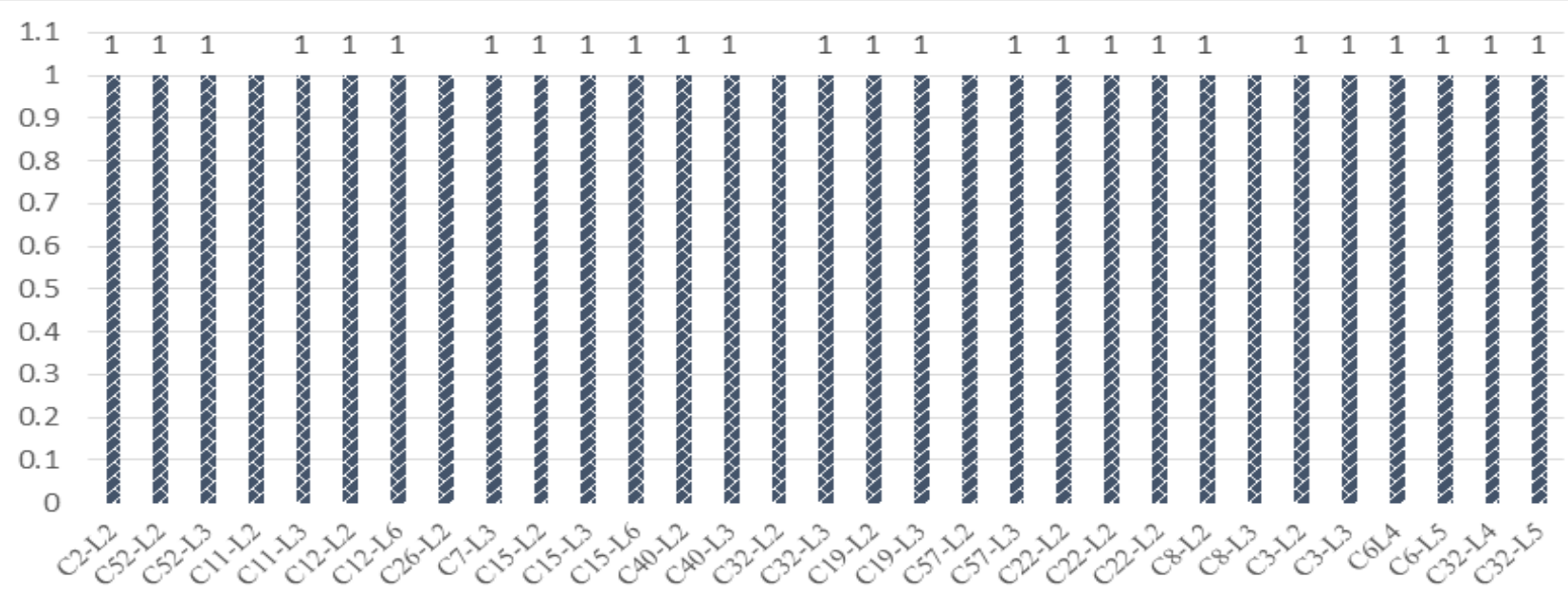

Fig. 10. The PKS and PNS Zones of perfect learner.

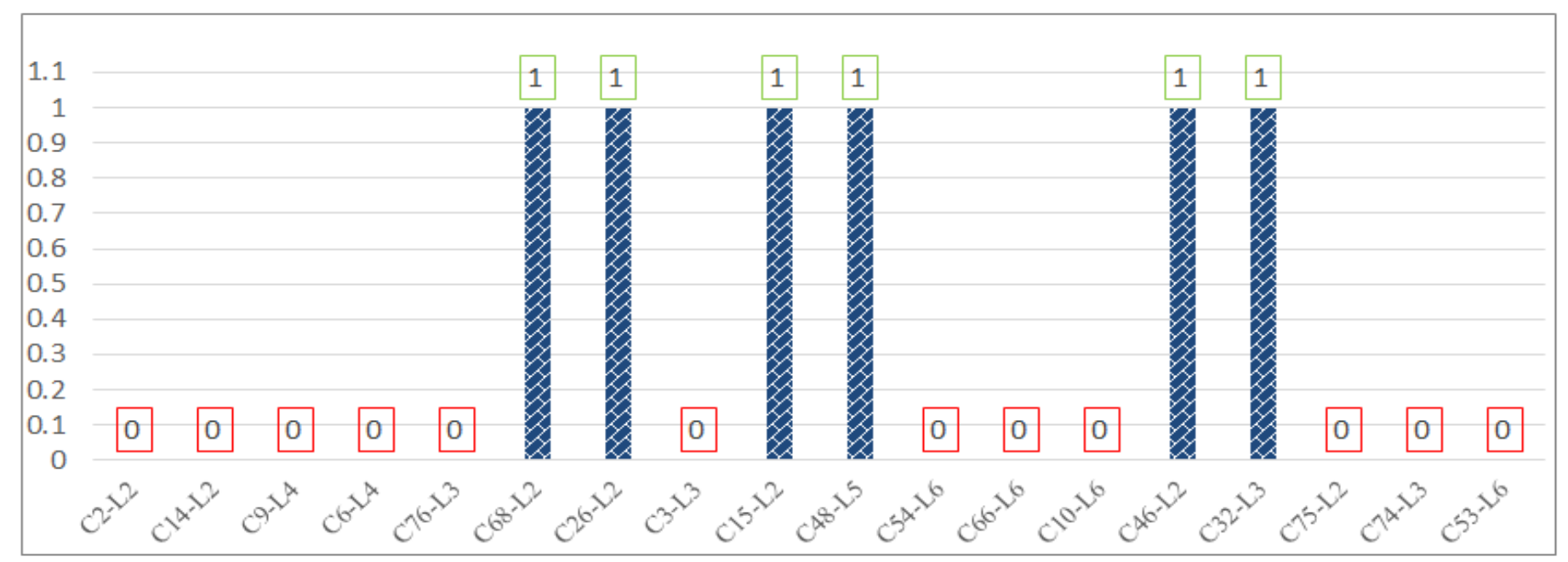

Fig. 11. The VKS and VNS Zones of learner \# 23.

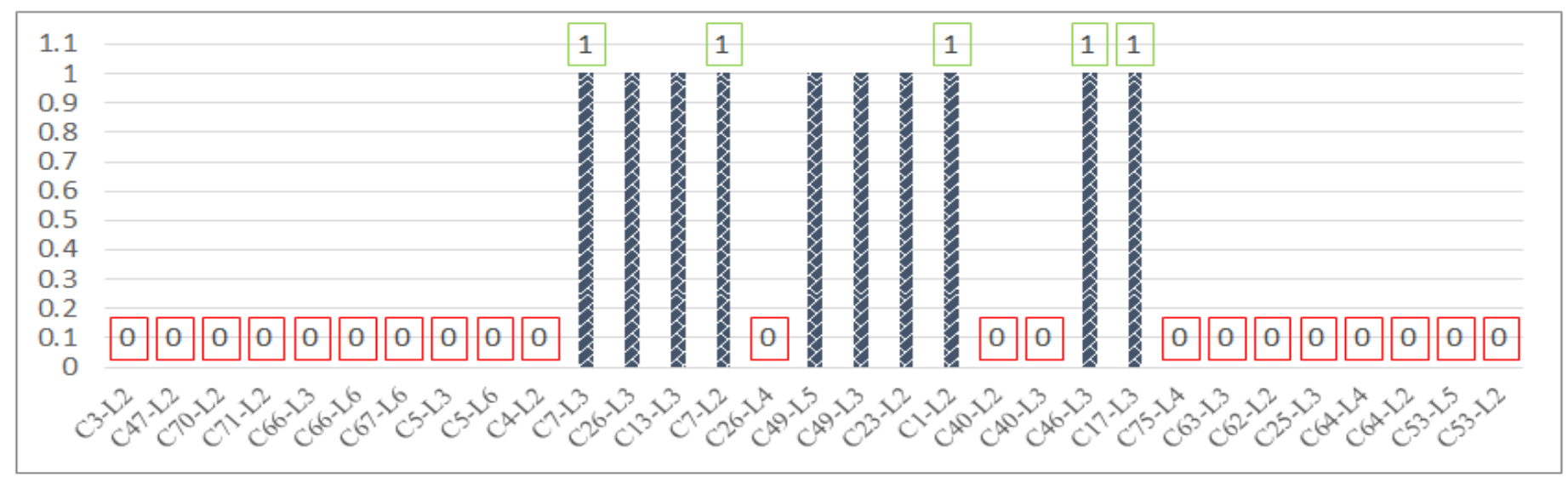

$\mathrm{X}$ : Concept Number

Y: Binary Number, "1" the concept is known. " 0 " the concept is unknown

Fig. 12. The DKS and DNS Zones of learner \# 23. 


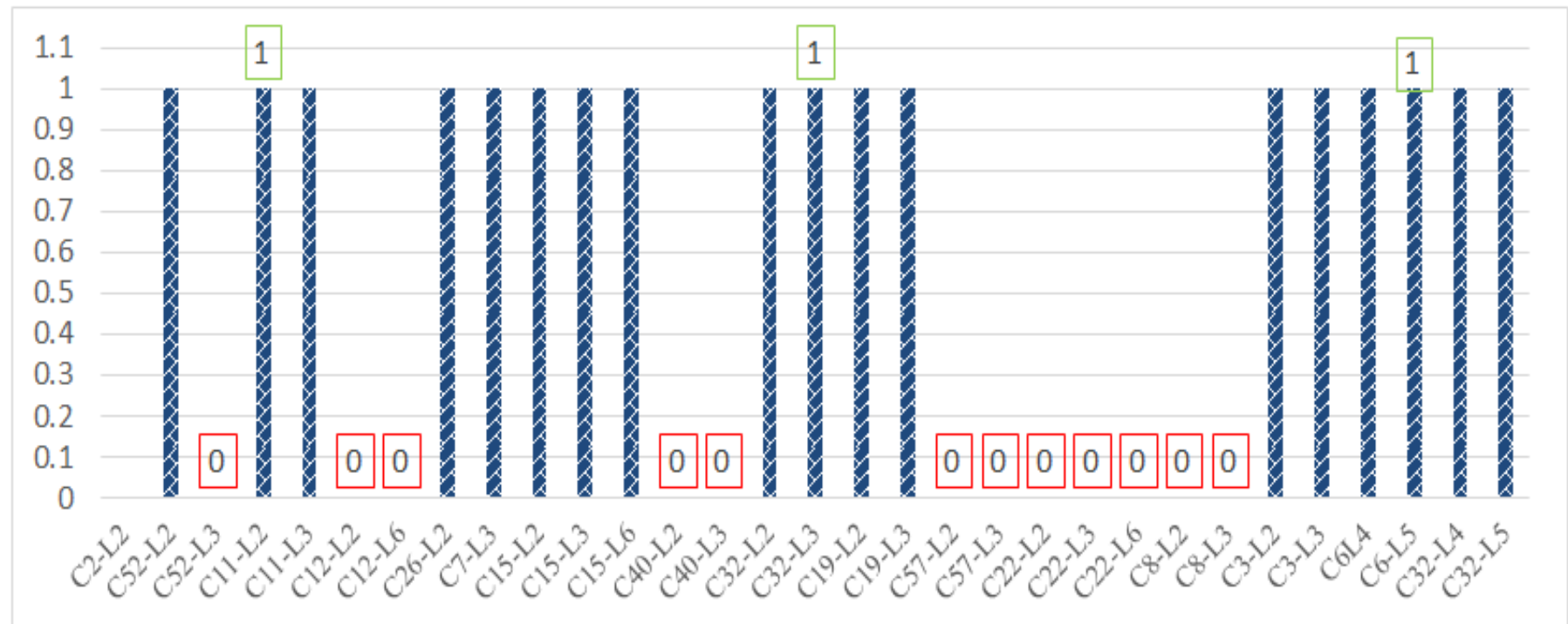
X: Concept Number
Y: Binary Number, "1" the concept is known. "0" the concept is unknown

Fig. 13. The PKS and PNS Zones of learner \# 23. 